\title{
Conocimiento y percepción sobre eutanasia en estudiantes de medicina de diecisiete países latinoamericanos, 2017
}

\author{
*Carlos Miguel Rios-González ${ }^{(1) a}$, Ginno Alessandro De Benedictis-Serrano(2)b, Génesys José Córdova- \\ Rivas $^{(2) b}$, María Lucía Contreras Romero ${ }^{(2) b}$, Laura Valentina Contreras Lugo ${ }^{(2) b}$ \\ ${ }^{1}$ Universidad Nihon Gakko, Dirección de Investigación. Fernando de la Mora, Paraguay \\ ${ }^{2}$ Universidad de Carabobo Sede Aragua, Facultad de Ciencias de la Salud. Venezuela
}

\begin{abstract}
Cómo referenciar este artículo/ How to reference this article:
\end{abstract}

\author{
Rios-González CM, De Benedictis-Serrano GA, \\ Córdova-Rivas GJ, Contreras Romero ML, \\ Contreras Lugo LV. Conocimiento y percepción sobre \\ eutanasia en estudiantes de medicina de diecisiete \\ países latinoamericanos, 2017. Mem. Inst. Investig. \\ Cienc. Salud. 2018; 16(3): 58-65
}

\section{R E S U M E N}

El objetivo fue determinar el conocimiento y la percepción de la eutanasia en los estudiantes de medicina de diecisiete países de Latinoamérica durante el 2017. Se realizó un estudio observacional descriptivo de corte transversal con componente analítico y con muestreo no probabilístico por conveniencia, el instrumento fue un cuestionario validado por expertos y una prueba piloto. Se utilizaron las medidas de tendencia central, dispersión y proporciones, para la comparación de los grupos se consideró una $p<0,05$ como significativa. Fueron incluidos 3924 estudiantes de medicina de 17 países latinoamericanos. El 69,05\%(2709) era de sexo femenino, el 93,91\%(3684) de los estudiantes conocía sobre la eutanasia, y el 70,97\% (2784) se encontraba a favor de la eutanasia. El 53,49\% (115) de los estudiantes con un rango etario de 20 a 24 años desconocía la eutanasia $(p=0,000)$, el 65,95\% (1836) de los estudiantes que estaba en el rango etario de 20 a 24 años estaba a favor de la eutanasia $(p=0,000)$, mientras que el $64 \%$ (576) de los de sexo femenino estaban en contra $(p=0,000)$. La gran mayoría de los estudiantes tenía conocimiento sobre la eutanasia y la mayor parte de los estudiantes tenían una percepción a favor. Existía una asociación estadísticamente significativa entre el conocimiento, la edad y los años de estudio, y a su vez una asociación estadísticamente significativa entre la percepción, la edad, el sexo y los años de estudio.

Palabras clave: eutanasia, estudiantes de medicina, discusiones bioéticas.

\section{Knowledge and perception about euthanasia in medical students from seventeen Latin American countries, 2017}

\section{A B S T R A C T}

The objective was to determine the knowledge and perception of euthanasia in medical students from seventeen Latin American countries during 2017. A cross-sectional descriptive observational study with an analytical component and non-probabilistic convenience sampling was carried out. The instrument was a questionnaire validated by experts and a pilot test. The measures of central tendency, dispersion and proportions were used for the comparison of the groups and a $p<0.05$ was considered as significant. Of the total number, 3,924 medical students from seventeen Latin American countries were included. The $69.05 \%(2,709)$ was female, $93.91 \%(3,684)$ of the students knew about euthanasia, and $70.97 \%(2,784)$ was in favor of euthanasia. The $53.49 \%(115)$ of the students with an age range of 20 to 24 years were unaware of euthanasia $(p=0.000)$, $65.95 \%(1,836)$ of the students with a range of ages of 20 to 24 years were in favor of euthanasia $(p=0.000)$, while $64 \%(576)$ of females were against it $(p=0.000)$. The 
majority of the students had knowledge about euthanasia and most students had a perception in favor. There was a statistically significant association between knowledge, age and years of study, and also a statistically significant association between perception, age, sex and years of study.

Keywords: euthanasia, medicine students, bioethical discussions.

\section{INTRODUCCIÓN}

Con el desarrollo social, la educación y la redistribución de las riquezas, y con la mejora de los cuidados médicos, se ha notado una variación en las causas de muerte, pero no en la muerte ${ }^{(1)}$, lo cual indica que llegará el momento en que todos se encuentren cara a cara con la muerte, porque ningún avance ha sido lo suficientemente efectivo para evitar la muerte ${ }^{(2)}$.

La medicina, a pesar de su progreso científico, continúa siendo una labor que no puede garantizar los resultados finales de la vida ${ }^{(3)}$, el objetivo médico a través de los años de acuerdo a la circunstancia ha cambiado su perspectiva de evitar, curar y aliviar a ayudar a morir con dignidad. Esto es un aspecto que obliga a considerar la exigencia de las limitaciones del esfuerzo diagnóstico y terapéutico, incluso la eutanasia(1). Se debe tomar en cuenta que aceptar la muerte como un hecho vital no es un fracaso, sin embargo, sí lo es aferrarse a prolongar la vida a toda costa; ayudar a morir en paz es tan importante como curar y cuidar $^{(4)}$. Cuando un paciente se halla en el período final de su vida, la prioridad médica no debería ser únicamente la conservación de la vida, sino preservar la calidad de esa vida que aún le queda ${ }^{(5)}$.

La eutanasia, etimológicamente, significa buena muerte, es decir, una manera ideal de morir, mientras que la Organización Mundial de la Salud (OMS) la define como la acción deliberada que realiza una persona con la intención de provocar la muerte sin dolor, a otro sujeto, o no prevenir la muerte por causa natural, en caso de enfermedad terminal o coma irreversible, este acto médico requiere, entonces, tres elementos imprescindibles: la petición expresa del enfermo, padecimiento físico o psíquico insoportable para el mismo, y una condición clínica terminal ${ }^{(6,7)}$.

Tradicionalmente, la nomenclatura internacional ha dividido el concepto de Eutanasia en activa y pasiva ${ }^{(7)}$ : la primera se refiere a la práctica de una acción médica con la que se acelera la muerte del paciente o se finaliza su vida, principalmente mediante fármacos que sedan y posteriormente generen un paro cardiorrespiratorio. Por otro lado, en la eutanasia pasiva no se induce la muerte, si no que se dejan de aplicar los tratamientos, medicamentos u operaciones que prolonguen la vida del paciente, como un respirador artificial o una sonda con alimentación parenteral. Asimismo, ambas clasificaciones pueden volver a dividirse en eutanasia voluntaria o involuntaria, caracterizándose la voluntaria por la solicitud por parte del paciente o un familiar, dependiendo de la ley de cada país en el que sea practicado este procedimiento, contrario a la involuntaria, donde el paciente no está en capacidades físicas o mentales de manifestar el deseo que la eutanasia sea aplicada, pero que previamente la solicitó, o puede presentarse también cuando un comité médico es el que toma la decisión de ejecutarla sin conocer cuál era la voluntad del paciente ${ }^{(8)}$.

En la actualidad, en cuatro países del mundo (Holanda, Bélgica, Luxemburgo, y Colombia) la eutanasia activa es legal, por otro lado hay numerosos países que permiten formalmente la eutanasia pasiva a través de sus regulaciones en diferentes formas (Canadá, España, Inglaterra, India, entre otros) ${ }^{(7)}$.

Se debe tener en cuenta que la muerte es una etapa de la vida, por lo tanto desear tener una buena muerte es una legítima aspiración de muchos seres humanos, es por ello, que los profesionales de la salud están obligados a ayudar a sus enfermos a que puedan tener una muerte digna, cuando no se ha conseguido prevenir ni curar su enfermedad ${ }^{(9)}$, lo cual sugiere una carga moral, psicológica y emocional para estos profesionales ${ }^{(10)}$ por lo que se debería pensar si los médicos comprenden cómo actuar y si están preparados para atender las situaciones y decisiones al final de la vida de sus pacientes ${ }^{(9,2)}$.

La capacitación profesional y la filosofía asistencial que han adquirido todos los profesionales del área de la salud incorporan la muerte como un fracaso, no incluyéndose en el período de formación de pre y postgrado, ni en los libros de texto, los argumentos docentes que los preparen para la hora de enfrentarse al tratamiento de los últimos momentos de la vida(2). 
Si bien, en la actualidad la investigación sobre este tema es amplia, y seguirá constituyendo un tema importante de investigación, puesto que representa uno de los dilemas éticos de la actualidad ${ }^{(11)}$.

Por todo lo anterior, se ha planteado como objetivo determinar el conocimiento y la percepción de la eutanasia en los estudiantes de medicina de diecisiete países de Latinoamérica durante el 2017.

\section{MATERIAL Y MÉTODOS}

Se realizó un estudio observacional, descriptivo de corte transversal, con componente analítico y con muestreo no probabilístico por conveniencia en estudiantes de medicina de Latinoamérica, durante los meses de agosto a septiembre de 2017.

Fueron incluidos 3924 estudiantes de la carrera de medicina de primero a octavo año pertenecientes a distintas universidades de Argentina, Bolivia, Brasil, Chile, Colombia, Ecuador, Guatemala, Hondura, México, Panamá, Paraguay, Nicaragua, Perú, Puerto Rico, República Dominicana, Uruguay y Venezuela, que accedieron a participar al estudio, otorgando su consentimiento informado.

A todos los participantes del estudio se les informó cuales eran los objetivos del estudio y aquellos que estuvieron de acuerdo, procedieron al llenado del cuestionario electrónico mediante Google Doc(C). Dicho cuestionario se publicó en redes sociales (Facebook@), Twitter( e Instagram (C), se envió por aplicación telefónica de mensajería instantánea (WhatsApp@) y a correos electrónicos.

Se diseñó un cuestionario, que contenía 35 preguntas, divididas en cuatro secciones: epidemiologia, preguntas generales, conocimientos y percepción. Se incluyeron cinco preguntas que determinaron las características epidemiológicas, siete preguntas generales que permiten discernir ciertas influencias durante la vida del estudiante que pueden afectar en el desarrollo del cuestionario al momento de responder, cinco preguntas de conocimiento que miden los niveles del estudiantes con respecto a la eutanasia y lo referente a la misma, en donde si el estudiante no tiene conocimientos de eutanasia el cuestionario se termina automáticamente, por último se incluyeron 18 preguntas que miden la percepción del estudiante ante la eutanasia.

Para la validación del instrumento, éste se envió a dos especialistas en bioética y se realizó un estudio piloto en 40 estudiantes de medicina de la Universidad de Carabobo Sede Aragua y la Universidad Nacional de Caaguazú, los cuales fueron excluidos de la muestra final, donde se realizó la evaluación y ajuste de cada uno de los constructos.

Para determinar el conocimiento se consideraron tres preguntas respondidas correctamente, mientras que para determinar la percepción se evaluó mediante una escala de tipo Likert donde se consideraron los ítems respondidos como Totalmente de acuerdo y de acuerdo como A favor, lo respondido con Indeciso/dudoso como neutral y los respondidos con En desacuerdo y Totalmente en desacuerdo como En contra.

Toda la información recogida por los investigadores fue tratada con confidencialidad, igualdad y justicia.

Todos los datos proveídos por los cuestionarios fueron cargados en Microsoft Office Excel $2013^{\circledR}$, y luego del control de calidad fueron exportados para su análisis en Stata $14.0^{\circledR}$. Los datos se expresan en medidas de tendencia central, dispersión y proporciones. Para el análisis de confiabilidad se obtuvo el coeficiente de alfa de Cronbach (valor mayor de 0,7 define una confiabilidad aceptable), para la comparación de los grupos y se consideró una $\mathrm{p}<0,05$ como significativa.

\section{RESULTADOS}

Durante los meses de agosto a septiembre del 2017 fueron incluidos un total de 3924 estudiantes de medicina, estando el 63,91 (2507) en un rango etario de 20 a 24 años de edad, el 69,05\% (2709) del sexo femenino. El 56.49\% $(2,216)$ de los participantes fueron de Venezuela, el 9,89\%(388) de Chile y el 7,24(284) de Argentina. La mayor participación de los encuestados fue de aquellos que se encontraban en los primeros años de la carrera, 3er. año con $22.43 \%$ (880) y 2 do.año con $21.92 \%$ (860), a su vez el $93,40 \%$ (3664) eran solteros y el $51,24 \%$ (2011) eran católicos (Tabla 1 ). 
Tabla 1: Distribución de los estudiantes de medicina de 17 países latinoamericanos de acuerdo a las características generales, 2017

\begin{tabular}{|c|c|c|}
\hline Características generales & $\mathbf{n}$ & $\%$ \\
\hline \multicolumn{3}{|l|}{ Edad } \\
\hline 18 a 19 años & 848 & 21.62 \\
\hline 20 a 24 años & 2,507 & 63.91 \\
\hline 25 a 29 años & 468 & 11.93 \\
\hline 30 a más años & 100 & 2.55 \\
\hline \multicolumn{3}{|l|}{ Sexo } \\
\hline Masculino & 1.214 & 30,95 \\
\hline Femenino & 2.709 & 69,05 \\
\hline \multicolumn{3}{|l|}{ País } \\
\hline Argentina & 284 & 7.24 \\
\hline Bolivia & 76 & 1.94 \\
\hline Brasil & 12 & 0.31 \\
\hline Chile & 388 & 9.89 \\
\hline Colombia & 36 & 0.92 \\
\hline Guatemala & 60 & 1.53 \\
\hline Honduras & 12 & 0.31 \\
\hline Ecuador & 180 & 4.59 \\
\hline México & 232 & 5.91 \\
\hline Nicaragua & 67 & 1.71 \\
\hline Panamá & 48 & 1.22 \\
\hline Paraguay & 108 & 2.75 \\
\hline Perú & 24 & 0.61 \\
\hline Puerto Rico & 156 & 3.98 \\
\hline Uruguay & 24 & 0.61 \\
\hline Venezuela & 2,216 & 56.49 \\
\hline \multicolumn{3}{|l|}{ Estado civil } \\
\hline Soltero & 3664 & 93,40 \\
\hline Casado & 175 & 4,46 \\
\hline Divorciado & 12 & 0,31 \\
\hline Unión libre & 72 & 1,84 \\
\hline \multicolumn{3}{|l|}{ Religión } \\
\hline Católica & 2011 & 51,24 \\
\hline Evangélica & 1021 & 26,01 \\
\hline Agnóstica & 410 & 10,44 \\
\hline Atea & 267 & 6,80 \\
\hline Otros & 215 & 5,47 \\
\hline \multicolumn{3}{|l|}{ Año cursando actualmente } \\
\hline $1^{\circ}$ año & 448 & 11.42 \\
\hline $2^{\circ}$ año & 860 & 21.92 \\
\hline $3^{\circ}$ año & 880 & 22.43 \\
\hline $4^{\circ}$ año & 768 & 19.58 \\
\hline $5^{\circ}$ año & 344 & 8.77 \\
\hline $6^{\circ}$ año & 379 & 9.66 \\
\hline $7^{\circ}$ año & 132 & 3.36 \\
\hline $8^{\circ}$ año & 112 & 2.85 \\
\hline
\end{tabular}

Del total de encuestados, el 93,91\% (3684) afirmó que si poseían los conocimientos adecuados sobre eutanasia mientras que el 6,09\% (239) no tenía conocimientos sobre el tema. En la escala estructurada para determinar la percepción de la eutanasia, se logró observar que el $70,97 \%$ (2784) se encontraba a favor y el $22,94 \%(900)$ se encontraba en contra (Tabla 2). 
Tabla 2: Distribución de los estudiantes de medicina de 17 países latinoamericanos de acuerdo al conocimiento y la percepción general sobre eutanasia, 2017

\begin{tabular}{lll}
\hline & $\mathrm{n}$ & $\%$ \\
\hline $\begin{array}{ll}\text { Conocimiento } \\
\text { Si }\end{array}$ & 3684 & 93,91 \\
$\quad$ No & 239 & 6,09 \\
Percepción & & \\
A favor & 2784 & 70,97 \\
Neutral & 0 & 0 \\
En contra & 900 & 22,94 \\
\hline
\end{tabular}

Los aspectos determinantes sobre como los estudiantes percibían la eutanasia se basaban en que el 53,09\% (1919) estaba en desacuerdo con que es un homicidio, el $45,30 \%$ (1669) en desacuerdo con que es un pecado, el 53,80\% (1982) en desacuerdo con que es inmoral y el $42,26 \%$ (1557) estaba de acuerdo con que es una muerte digna (Tabla $3)$.

Tabla 3: Distribución de los estudiantes de medicina de 17 países latinoamericanos de acuerdo a los aspectos determinantes sobre la percepción de la eutanasia, 2017

\begin{tabular}{|c|c|c|c|c|c|c|c|c|c|c|}
\hline \multirow[t]{2}{*}{$\begin{array}{l}\text { Eutanasia } \\
\text { es: }\end{array}$} & \multicolumn{2}{|c|}{$\begin{array}{l}\text { Totalmen } \\
\text { te de } \\
\text { acuerdo }\end{array}$} & \multicolumn{2}{|c|}{ De acuerdo } & \multicolumn{2}{|c|}{ Indeciso } & \multicolumn{2}{|c|}{ En desacuerdo } & \multicolumn{2}{|c|}{$\begin{array}{l}\text { Totalmente en } \\
\text { desacuerdo }\end{array}$} \\
\hline & $\mathbf{n}$ & $\%$ & $\mathbf{n}$ & $\%$ & $\mathrm{n}$ & $\%$ & $\mathrm{n}$ & $\%$ & $\mathrm{n}$ & $\%$ \\
\hline Homicidio & 152 & 4,13 & 244 & 6,62 & 0 & 0 & 1919 & 52,09 & 1369 & 37,16 \\
\hline Pecado & 340 & 9,23 & 368 & 9,99 & 0 & 0 & 1669 & 45,30 & 1307 & 35,48 \\
\hline Inmoral & 148 & 4,02 & 276 & 7,49 & 0 & 0 & 1982 & 53,80 & 1278 & 34,69 \\
\hline Muerte digna & 647 & 17,56 & 1557 & 42,26 & 0 & 0 & 1204 & 32,68 & 276 & 7,49 \\
\hline
\end{tabular}

El 53,49\% (115) de los estudiantes con un rango etario de 20 a 24 años desconocía la eutanasia $(p=0,000)$, el $66,98 \%(144)$ del sexo femenino desconocía $(p=0,539)$ así como el $34,88 \%$ (75) de los que estaban cursando el 4to. año $(p=0,000)$ (Tabla 4). Respecto a la percepción sobre la eutanasia, el $65,95 \%$ (1836) de los estudiantes con un rango etario de 20 a 24 años estaba a favor $(p=0,000)$, el 64\% (576) de los de sexo femenino estaban en contra $(p=0,000)$, mientras que el $25,33 \%$ (228) de los de 3er. año estaban en contra $(p=0,000)$ (Tabla 5).

Tabla 4: Comparación del conocimiento sobre eutanasia y las características generales de los estudiantes de medicina de 17 países latinoamericanos, 2017

\begin{tabular}{|c|c|c|c|c|}
\hline & & $\mathbf{S i}$ & & No \\
\hline & $\mathbf{n}$ & $\%$ & $\mathbf{n}$ & $\%$ \\
\hline \multicolumn{5}{|l|}{ Edad } \\
\hline 18 a 19 años & 748 & 20,30 & 100 & 46,51 \\
\hline 20 a 24 años & 2368 & 64,28 & 115 & 53,49 \\
\hline 25 a 29 años & 468 & 12,70 & 0 & 0 \\
\hline \multirow{2}{*}{30 a más años } & 100 & 2,71 & 0 & 0 \\
\hline & \multicolumn{4}{|c|}{ Pearson chi2 $(3)=101.0153 \quad P=0.000$} \\
\hline \multicolumn{5}{|c|}{ 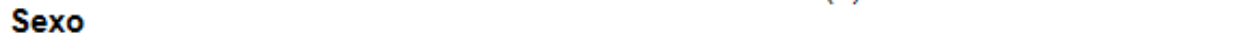 } \\
\hline Masculino & 1143 & 31,03 & 71 & 33,02 \\
\hline \multirow[t]{2}{*}{ Femenino } & 2541 & 68,97 & 144 & 66,98 \\
\hline & \multicolumn{4}{|c|}{ Pearson chi2 $(1)=0.3779 \quad P=0.539$} \\
\hline \multicolumn{5}{|c|}{ Años de estudio } \\
\hline 1 año & 412 & 11,18 & 36 & 16,74 \\
\hline 2 año & 792 & 21,50 & 68 & 31,63 \\
\hline 3 año & 844 & 22,91 & 36 & 16,74 \\
\hline 4 año & 669 & 18,16 & 75 & 34,88 \\
\hline 5 año & 344 & 9,34 & 0 & 0 \\
\hline 6 año & 379 & 10,29 & 0 & 0 \\
\hline 7 año & 132 & 3,58 & 0 & 0 \\
\hline \multirow[t]{2}{*}{8 año } & 112 & 3,04 & 0 & 0 \\
\hline & \multicolumn{4}{|c|}{ Pearson chi2 $(7)=104.5496 \quad P=0.000$} \\
\hline
\end{tabular}


Tabla 5: Comparación de la percepción sobre eutanasia y las características generales de los estudiantes de medicina de 17 países latinoamericanos, 2017

\begin{tabular}{|c|c|c|c|c|}
\hline & \multicolumn{2}{|c|}{ A favor } & \multicolumn{2}{|c|}{ En contra } \\
\hline & $\mathbf{n}$ & $\%$ & $\mathbf{n}$ & $\%$ \\
\hline \multicolumn{5}{|l|}{ Edad } \\
\hline 18 a 19 años & 528 & 18,97 & 220 & 24,44 \\
\hline 20 a 24 años & 1836 & 65,95 & 532 & 59,11 \\
\hline 25 a 29 años & 368 & 13,22 & 100 & 11,11 \\
\hline 30 a más años & & 1,87 & 48 & 5,33 \\
\hline & Pears & $(3)=47.4710$ & $P=0.000$ & \\
\hline \multicolumn{5}{|c|}{ 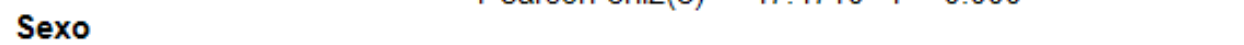 } \\
\hline Masculino & 819 & 29,42 & 324 & 36,00 \\
\hline \multirow{2}{*}{ Femenino } & 1965 & 70,58 & 576 & 64,00 \\
\hline \multirow{2}{*}{\multicolumn{5}{|c|}{ Pearson chi2 $(1)=13.7684 \quad P=0.000$}} \\
\hline & & & & \\
\hline 1 año & 332 & 11,93 & 80 & 8,89 \\
\hline 2 aก̃o & 580 & 20,83 & 212 & 23,56 \\
\hline 3 aก̃o & 616 & 22,13 & 228 & 25,33 \\
\hline 4 año & 541 & 19,43 & 128 & 14,22 \\
\hline 5 aก̃o & 200 & 7,18 & 144 & 16,00 \\
\hline 6 año & 331 & 11,89 & 48 & 5,33 \\
\hline 7 año & 120 & 4,31 & 12 & 1,33 \\
\hline \multirow[t]{2}{*}{8 año } & 64 & 2,30 & 48 & 5,33 \\
\hline & Pears & $(7)=143.6215$ & $P=0.000$ & \\
\hline
\end{tabular}

\section{DISCUSIÓN}

El conflicto moral sobre el tema de la eutanasia siempre existirá, por lo que en varios países del mundo se desarrollan iniciativas de regulación del derecho a decidir al final de la vida, teniendo en cuenta que es posible comenzar por una ética mínima que logre optimizar la atención al final de la vida, evitando situaciones de incertidumbre que pueden vivir los pacientes y sus familiares, e incluso el personal médico ${ }^{(12)}$. En la actualidad escuchar la palabra eutanasia confiere un fuerte componente emocional, debido a los múltiples sentidos en los que es utilizada, teniendo una connotación negativa por la carga emotiva. Se tiende a utilizar neologismos como distanasia, ortotanasia o cacotanasia, también se adjetiva en directa-indirecta, voluntaria-involuntaria o activa-pasiva; esto lleva a incrementar la confusión terminológica debido a distintas palabras y oraciones que llevan al mismo sentido que es "el buen morir" que es lo que se refiere etimológicamente la eutanasia ${ }^{(13)}$.

Este trabajo logro demostrar que los estudiantes de medicina poseen conocimientos adecuados en eutanasia y están a favor de la misma lo cual no es ningún riesgo para la sociedad actual ya que en Holanda, país que posee leyes que aprueban la eutanasia, se han realizado investigaciones entre 1990 y 2010, en donde lograron determinar la estabilidad en el perfil de los pacientes que solicitan la eutanasia, pero no demostraron un incremento significativo en el número de casos solo por ser legal ${ }^{(14)}$.

Estos resultados muestran similitud con estudios realizados en Perú(15) y Venezuela ${ }^{(16)}$, pero contrastan con otros realizados en México ${ }^{(17)}$ y Alemania $^{(18)}$, donde el porcentaje a favor de la eutanasia es menor, en la población general, aunque en estos últimos años se ha producido un aumento en el apoyo a la eutanasia ${ }^{(11)}$, esta diferencia se podría deber a que se considera un hecho punible en dichos países, además en México la alta proporción de religiosidad.

En el estudio se puede apreciar además los de sexo masculino se encuentran mayoritariamente a favor, así como los de mayor edad y con más años de estudio, siendo esta diferencia estadísticamente significativa.

El desconocimiento, la falta de autoeducación, los mitos, las actitudes negativas, las leyes incompletas y los obstáculos religiosos son algunos de los impedimentos que hacen que la eutanasia se perciba como un resultado desfavorable. Se debe romper el paradigma y aún más en Latinoamérica.

Es importante para autoridades públicas, maestros, profesionales y estudiantes de las ramas de la salud tomar en cuenta los resultados de este estudio para concientizar y buscar estrategias que soluciones este dilema socio cultural y de formación profesional. 
Es crucial el rol que los profesores pueden tener en los estudiantes de medicina tanto en promover las investigaciones en temas de bioética y específicamente en eutanasia ${ }^{(19)}$ o simplemente llevar a que el estudiante emprenda un razonamiento lógico sobre la eutanasia. Se deben realizar debates y mesas de trabajo que favorezcan el entendimiento en los estudiantes y profesionales de la salud que lleven a una mejor comprensión del paciente que adolece gravemente, por lo que se puede cambiar el mundo desde la formación educativa en ciencias de la salud.

Es difícil evaluar una práctica que es prohibida en el entorno, por lo que las preguntas realizadas no condicionaban la práctica de la eutanasia en un marco legal, lo que trae a discusión si la práctica fuese permitida sería una gran cantidad de futuros médicos quienes podrían practicarla adecuadamente con sus pacientes.

Las limitaciones el estudio se centra en el muestreo, siendo un muestreo no probabilístico presenta limitaciones en la validez externa, no pudiendo generalizarse los resultados de este estudio además de que no se pudo acceder a la totalidad de los países latinoamericanos.

En conclusión, una importante proporción de los estudiantes tiene conocimiento sobre la eutanasia y la percepción es a favor en su mayoría, asimismo existe una asociación estadísticamente significativa entre el conocimiento, la edad y los años de estudio, a su vez la percepción con la edad, el sexo y los años de estudio, por lo que es necesario conducir nuevos estudios que conformen grupos interdisciplinarios para iniciar el dialogo sobre la eutanasia en todos los países.

Financiado con fondos propios de los autores.

Los autores no declaran ningún conflicto de interés.

\section{REFERENCIAS BIBLIOGRÁFICAS}

1. Gervas J. Morir en casa con dignidad. Una posibilidad, si hay apoyo y cuidados de calidad. Gaceta Médica de Bilbao. 2011; 108(1):3-6.

2. Kessel Sardinas $H$. Paradojas en las decisiones al final de la vida. MEDICINA CLÍNICA. 2001; 116 (8): 296-8.

3. García Otero Al, Sabio MF. ¿Existe justificación moral para la eutanasia?. Rev Am Med Resp. 2011; 11(4): 202-9.

4. Sarabia Alvarezudea J. Atención a los pacientes con enfermedades en fase terminal: la opinión de los ciudadanos. Medicina Clínica (Barc). 2012; 138(2):73-7.

5. Casas Martinez ML. Sedación terminal, eutanasia y bioética. Revista Cubana de Medicina. 2005;44: 5-6.

6. Mendoza-Villa JM, Herrera-Morales LA. Reflexiones acerca de la eutanasia en Colombia. Rev Colomb Anestesiol. 2016; 44(4): 324-9.

7. Carrasco M VH, Crispi F. Eutanasia en Chile: Una discusión pendiente. Rev Med Chile. 2016; 144(12): 1598-604.

8. Hurtado Medina MJ. La eutanasia en Colombia desde una perspectiva bioética. Revista Médica de Risaralda. 2016; 22 (1): 4951.

9. Batiz J. Ayudar a morir bien. Gaceta Médica de Bilbao. 2007; 104(3): 97

10. Goic GA. Apuntes sobre la eutanasia. Rev Méd Chile. 2005; 133(3): 371-5.

11. Rios-González CM, De Benedictis-Serrano GA, Contreras Lugo LV, Córdova-Rivas GJ, Contreras Romero ML. Análisis bibliométrico sobre las publicaciones de eutanasia en SCOPUS, MEDLINE/Pubmed, Science Citation Index y SciELO. Rev Med Hered. 2018; 29(2): 125-6.
12. Melguizo Jiménez $M$, Lorda PS, Arriba Marcos B.Eutanasia y suicidio asistido. AMF. 2015;11(7):384-91

13. Lorda PS, Barrio IM, Alarcos FJ, Barbero J, Couceiro A, Hernando P. Ética y muerte digna: propuesta de consenso sobre un uso correcto de las palabras. Revista de Calidad Asistencial. 2008;23 (6);271-85

14. Onwuteaka-Philipsen BD, BrinkmanStoppelenburg A, Penning C, De Jong-Krul GJ, van Delden JJ, Van der Heide A. Trends in endoflife practices before and after the enactment of the euthanasia law in the Netherlands from 1990 to 2010: a repeated cross-sectional survey. Lancet. 2012;380 (9845):908-15.

15. García Hirsch SMI, Guevara Peralta RJ. Percepción de la Eutanasia y factores asociados, en estudiantes de Medicina de la Facultad de Ciencias de la Salud de la Universidad Peruana de Ciencias. 2014 [acceso 30 de Diciembre de 2017]; Disponible en: http://repositorioacademico.upc.edu.pe/upc/ha ndle/10757/322312

16. Anneser J, Kunath N, Krautheim V, Borasio GD. Needs, expectations, and concerns of medical students regarding end-of-life issues before the introduction of a mandatory undergraduate palliative care curriculum. J Palliat Med. 2014;17(11):1201-5.

17. Anneser J, Jox R, Borasio GD. Physicianassisted suicide, euthanasia and palliative sedation: attitudes and knowledge of medical students. GMS J Med Educ . 2016;33(1):1-14. 18. Ramirez-Rivera J, Cruz J, Jaume-Anselmi F. Euthanasia, assisted suicide and end-of-life care: attitudes of students, residents and 
attending physicians. P R Health Sci J. 2006;25(4):325-29.

19. Marco FF. Voluntades vitales anticipadas: el reto de desarrollar un papel desde atención primaria. Gac Sanit. 2008;40(2):67-8.
20. Ríos González CM, De Benedictis-Serrano G, D’Amico-López R. El rol docente en la promoción de la investigación científica en pregrado. Educ Med. 2017. Doi: 10.1016/j.edumed.2017.07.021. 